\title{
Does the method of radiologic surveillance affect survival after resection of stage I non-small cell lung cancer?
}

\author{
Traves D. Crabtree, MD, ${ }^{a}$ Varun Puri, MD, ${ }^{a}$ Simon B. Chen, BS, ${ }^{b}$ David S. Gierada, MD, \\ Jennifer M. Bell, BSN, ${ }^{\text {a }}$ Stephen Broderick, MD, ${ }^{a}$ A. Sasha Krupnick, MD, ${ }^{a}$ Daniel Kreisel, MD, PhD, ${ }^{a}$ \\ G. Alexander Patterson, MD, ${ }^{\mathrm{a}}$ and Bryan F. Meyers, $\mathrm{MD}, \mathrm{MPH}^{\mathrm{a}}$
}

\begin{abstract}
Objectives: Controversy persists regarding appropriate radiographic surveillance strategies after lung cancer resection. We compared the impact of surveillance computed tomography scan versus chest radiography in patients who underwent resection for stage I lung cancer.
\end{abstract}

\begin{abstract}
Methods: A retrospective analysis was performed of all patients undergoing resection for pathologic stage I lung cancer from January 2000 to April 2013. After resection, follow-up included routine history and physical examination in conjunction with chest radiography or computed tomography at the discretion of the treating physician. Identification of successive lung malignancy (ie, recurrence at any new site or new primary) and survival were recorded.
\end{abstract}
Results: There were 554 evaluable patients, with 232 receiving routine postoperative computed tomography and 322 receiving routine chest radiography. Postoperative 5-year survival was $67.8 \%$ in the computed tomography group versus $74.8 \%$ in the chest radiography group $(P=.603)$. Successive lung malignancy was found in $27 \%$ (63/232) of patients receiving computed tomography versus $22 \%$ (72/322) receiving chest radiography $(P=.19)$. The mean time from surgery to diagnosis of successive malignancy was 1.93 years for computed tomography versus 2.56 years for chest radiography $(P=.046)$. For the computed tomography group, $41 \%$ (26/63) of successive malignancies were treated with curative intent versus $40 \%$ (29/72) in the chest radiography group $(P=.639)$. Cox proportional hazard analysis indicated imaging modality (computed tomography vs chest radiography) was not associated with survival $(P=.958)$.

Conclusions: Surveillance computed tomography may result in earlier diagnosis of successive malignancy versus chest radiography in stage I lung cancer, although no difference in survival was demonstrated. A randomized trial would help determine the impact of postoperative surveillance strategies on survival. (J Thorac Cardiovasc Surg 2015; 149:45-53)

See related commentary on pages 53-4.

Supplemental material is available online.

From the Division of Cardiothoracic Surgery, ${ }^{\mathrm{a}}$ Department of Surgery, Washington University School of Medicine, St Louis, Mo; Washington University School of Medicine, ${ }^{\mathrm{b}}$ St Louis, Mo; and Department of Radiology, ${ }^{\mathrm{c}}$ Washington University School of Medicine, St Louis, Mo.

Disclosures: Traves D. Crabtree reports consulting fees for Ethicon. Bryan F. Meyers reports consulting fees for Ethicon and Varian Medical Systems. All other authors have nothing to disclose with regard to commercial support.

Read at the 94th Annual Meeting of The American Association for Thoracic Surgery, Toronto, Ontario, Canada, April 26-30, 2014.

Received for publication April 9, 2014; revisions received July 28, 2014; accepted for publication July 31, 2014; available ahead of print Sept 11, 2014.

Address for reprints: Traves D. Crabtree, MD, Cardiothoracic Surgery, Barnes-Jewish Hospital, 3108 Queeny Tower, One Barnes-Jewish Hospital Plaza, St Louis, MO 63110 (E-mail: crabtreet@wudosis.wustl.edu).

$0022-5223 / \$ 36.00$

Copyright $\odot 2015$ by The American Association for Thoracic Surgery

http://dx.doi.org/10.1016/j.jtcvs.2014.07.095
Currently, there is no consensus on the optimal method of radiographic follow-up after resection of stage I non-small cell lung cancer (NSCLC). Patients undergoing resection for pathologic stage I NSCLC are still at risk of developing recurrence and ${ }^{1-4}$ developing a new primary lung cancer at an estimated rate of 1 to 6 cases per 100 person-years. ${ }^{5-9}$ The risk of identifying lung cancer in a high-risk group of smokers in the National Lung Screening Trial (NLST) was approximately $0.6 \%$ per person-year. ${ }^{10}$ Because of the substantial combined risk of recurrence and development of new primary lung cancers, multiple guidelines have been recommended for routine postoperative radiographic surveillance after resection for NSCLC (Table E1). ${ }^{9,11-13}$

Recently published results from the NLST demonstrated that routine low-dose computed tomography (CT) scans of patients at high risk for developing a first primary lung cancer offered a lung cancer-specific survival benefit compared with routine chest radiograph. However, it is unclear what impact radiographic surveillance will have on survivors of resected NSCLC, who are subject to competing risks of recurrence and new primary lung cancer. 


$\begin{aligned} & \text { Abbreviations and Acronyms } \\ & \text { CONSORT }= \text { Consolidated Standards of Reporting } \\ & \text { Trials } \\ & \text { CT }=\text { computed tomography } \\ & \text { CXR }=\text { chest } x \text {-ray } \\ & \text { NLST }=\text { National Lung Screening Trial } \\ & \text { NSCLC }=\text { non-small cell lung cancer } \\ & \text { PET }=\text { positron emission tomography }\end{aligned}$

Although CT has evolved as the standard for routine postoperative surveillance, there remains a paucity of data demonstrating a distinct survival advantage for CT over less-expensive imaging follow-up. A recent single-arm prospective trial suggested that minimal-dose CT may be beneficial in the postoperative surveillance of patients with stage I to III NSCLC. ${ }^{14}$ Historically, however, routine postoperative CT surveillance has resulted in inconsistent results regarding earlier detection of recurrence or new primary lung cancer, with no conclusive evidence of improvement in survival (Table E2). ${ }^{4-6,14-20}$

We performed a comparison of recurrence patterns and survival of patients with resected stage I NSCLC who underwent routine $\mathrm{CT}$ versus chest $\mathrm{x}$-ray (CXR) for postoperative surveillance. Our objective was to determine whether routine CT imaging led to earlier diagnosis of recurrence or new primary lung cancer and, if so, whether early detection translated into a survival benefit. Our primary hypothesis was that CT surveillance would result in earlier detection and secondarily that earlier detection would increase survival.

\section{PATIENTS AND METHODS}

All patients treated for pathologic stage I (T1-T2a N0 M0) NSCLC by pulmonary resection between January 2000 and April 2013 at Barnes-Jewish Hospital were identified from a prospective database and retrospectively analyzed in accordance with a protocol approved by the institutional review board at the Washington University School of Medicine. Patients were staged using the seventh edition of the lung cancer TNM staging system. Initial exclusion criteria included (1) malignancy within 5 years before resection (except squamous and basal cell skin carcinoma or carcinoma in situ) or a patient with contralateral nodules at the time of resection of the index lesion; (2) induction therapy; (3) incomplete resection; (4) missing surveillance records; (5) variability in surveillance imaging modality; (6) clinically detected recurrence before initiation of surveillance; (7) death before initiation of surveillance; and (8) age less than 18 years. Figure E1 shows a Consolidated Standards of Reporting Trials (CONSORT) diagram of the patient selection.

\section{Surveillance}

Each patient had an initial postoperative visit with the treating surgeon, consisting of a physical examination and a CXR, 2 to 4 weeks after resection. Subsequently, patients were followed by routine visits that consisted of a thorough history and physical examination and either CXR or CT scan. The imaging modality was chosen at the discretion of the treating physician. Certain surgeons consistently used CXRs for surveillance, whereas others initially used CXR and then transitioned to regimens that included a mixture of CXRs and CT or CT only. Some surgeons who used CT initially transitioned to a combination of CXR and CT. Surgeon "intent" could not be reliably determined for this subset of patients; thus, they were not included in the final analysis. In a separate analysis, there was no association between the treating surgeon and the overall survival (Figure E2). CXRs tended to be used more in the early period of this study. The mean date of resection for patients undergoing routine CT surveillance was May 15, 2009, and June 1, 2005, for CXR $(P<.001)$. CT scans included sections of the thorax and upper abdomen (including liver and adrenal glands) and were done with or without contrast, although the default was low-dose noncontrast CT. Generally, the standard follow-up regimen included a baseline CT scan approximately 3 months after resection, with follow-up imaging every 6 months for the first 2 to 3 years and then annually thereafter for at least 5 years. Standard CT technique included 5-mm contiguous sections until 2009 and 3-mm contiguous sections thereafter. Surveillance was defined as routine follow-up of asymptomatic patients after the initial postoperative visit for screening for recurrence or new primary lung cancer. For this study, patients were categorized in the CT group if they had routine CT scans or in the CXR group if they had routine CXR for surveillance. To minimize heterogeneity of follow-up, patients were excluded from the study if (1) the modality of their surveillance imaging switched because of changes in attending physician or in surveillance protocol for reasons not related to suspicion of recurrence; (2) they alternated between CXR and CT; or (3) they had routine positron emission tomography (PET) scans. False-positive surveillance scans resulting in invasive procedures, including bronchoscopy, needle biopsy, and surgical resection, were noted.

\section{Patient and Surveillance Characteristics}

There were 1140 patients who underwent resection for pathologic stage I lung cancer. Of these, 586 patients were excluded for malignancy within the previous 5 years $(\mathrm{n}=217)$, induction therapy $(\mathrm{n}=21)$, incomplete resection $(n=16)$, missing surveillance records, including those who followed up with an outside physician without accessible records $(\mathrm{n}=163)$, inconsistency in imaging modality used for surveillance or routine PET imaging $(n=149)$, recurrence before initiation of surveillance $(n=7)$, expiration before initiation of surveillance $(n=12)$, and age less than 18 years of age at the time of treatment $(n=1)$. Of the 149 patients excluded for inconsistency in imaging method, 123 switched the method because of a change in follow-up physician or protocol, 22 alternated between CT and CXR surveillance, 3 had routine CXR surveillance but had their successive malignancy detected by imaging obtained for unrelated issues, and 1 had routine PET imaging.

Among the 554 patients included, 232 underwent routine surveillance with CT and 322 underwent routine surveillance with CXR (Table 1). The median duration of follow-up up was 2.5 years (range, 0.3-9.9 years) for the CT group and 3.5 years (range, 0.1-13.1 years) for the CXR group. There were no significant differences between the groups in most baseline characteristics, with the exception of pathologic T stage of index tumor, type of surgical resection, and adjuvant therapy (Table 1).

\section{Successive Malignancy and Survival}

Successive malignancy was defined as recurrence or new primary lung cancer. The Martini and Melamed's criteria ${ }^{3}$ were used to identify new primary lung cancers as lesions that were (1) of different histology than the index cancer; (2) found in a different lobe, with no evidence of extrapulmonary or lymphatic metastasis; or (3) found at least 2 years after the index cancer. Successive malignancies were categorized according to whether they were detected during a scheduled or unscheduled visit, the presence of relevant symptoms at time of detection, and the imaging modality responsible for detection. Relevant symptoms included dyspnea or cough, pleuritic chest pain, hemoptysis, pneumonia, pneumothorax, hoarseness, weight loss, musculoskeletal pain, subcutaneous mass or 
TABLE 1. Baseline characteristics and mean number of follow-up imagings performed for computed tomography and chest $x$-ray groups

\begin{tabular}{|c|c|c|c|}
\hline Characteristic & $\begin{array}{c}\text { CT } \\
(\mathbf{n}=\mathbf{2 3 2}) \\
\end{array}$ & $\begin{array}{c}\text { CXR } \\
(\mathbf{n}=\mathbf{3 2 2})\end{array}$ & $\begin{array}{c}P \\
\text { value }\end{array}$ \\
\hline Male gender & $101(44 \%)$ & $121(38 \%)$ & .158 \\
\hline Caucasian race & $211(91 \%)$ & $283(88 \%)$ & .253 \\
\hline Age $($ mean \pm SD) & $65 \pm 11$ & $66 \pm 11$ & .346 \\
\hline $\begin{array}{l}\text { Charlson index (preoperative) } \\
\quad(\text { mean } \pm \mathrm{SD})\end{array}$ & $0.91 \pm 0.94$ & $0.91 \pm 1.08$ & .927 \\
\hline Tumor size $(\mathrm{cm})($ mean $\pm \mathrm{SD})$ & $2.3 \pm 1.1$ & $2.4 \pm 1.1$ & .332 \\
\hline T2a tumor & $94(41 \%)$ & $103(32 \%)$ & .039 \\
\hline Tumor histology & & & .366 \\
\hline Adenocarcinoma & $128(55 \%)$ & $159(49 \%)$ & \\
\hline Bronchoalveolar & $24(10 \%)$ & $28(9 \%)$ & \\
\hline Squamous & $41(18 \%)$ & $69(21 \%)$ & \\
\hline Adenosquamous & $5(2 \%)$ & $8(2 \%)$ & \\
\hline Carcinoid & $20(9 \%)$ & $43(13 \%)$ & \\
\hline Other or mixed & $14(6 \%)$ & $15(5 \%)$ & \\
\hline Resection procedure & & & .002 \\
\hline Wedge & $46(20 \%)$ & $27(8 \%)$ & \\
\hline Segmentectomy & $17(7 \%)$ & $19(6 \%)$ & \\
\hline Lobectomy & $152(66 \%)$ & $260(81 \%)$ & \\
\hline Sleeve & $7(3 \%)$ & $6(2 \%)$ & \\
\hline Bilobectomy & $4(2 \%)$ & $4(1 \%)$ & \\
\hline Wedge and lobectomy & $5(2 \%)$ & $3(1 \%)$ & \\
\hline Pneumonectomy & $1(0.4 \%)$ & $3(1 \%)$ & \\
\hline Adjuvant therapy & $48(21 \%)$ & $11(3 \%)$ & $<.001$ \\
\hline Chemotherapy & $39(17 \%)$ & $7(2 \%)$ & \\
\hline Radiation & $8(3 \%)$ & $3(1 \%)$ & \\
\hline Chemoradiation & $1(0.4 \%)$ & $1(0.3 \%)$ & \\
\hline \multicolumn{4}{|l|}{$\begin{array}{c}\text { No. of follow-up CT or CXR } \\
\text { per year }(\text { mean } \pm \text { SD) }\end{array}$} \\
\hline First year & $1.74 \pm 0.81$ & $2.12 \pm 0.86$ & $<.001$ \\
\hline Second year & $1.74 \pm 0.83$ & $1.66 \pm 0.72$ & .310 \\
\hline After second year & $1.34 \pm 0.55$ & $1.25 \pm 0.47$ & .122 \\
\hline Overall & $1.56 \pm 0.51$ & $1.58 \pm 0.56$ & .605 \\
\hline
\end{tabular}

$C T$, Computed tomography; $C X R$, chest x-ray; $S D$, standard deviation.

swelling, or neurologic changes. Also included were all new-onset symptoms noted in the records by the physician as suspicious for recurrence or warranting further imaging and symptoms that prompted an unscheduled visit in which recurrence or new primary was subsequently diagnosed. For treatment of successive malignancies, therapy was defined as "curative intent" when it included surgical resection or stereotactic radiation therapy for a localized pulmonary lesion or solitary brain or adrenal metastasis, or definitive chemoradiation for locoregional disease, such as an isolated recurrence in mediastinal lymph nodes. "Palliative therapy" was defined as chemotherapy alone, radiation therapy for symptom management, or pain management.

Overall survival was defined as starting from the date of resection for the index lung cancer and ending on the date of expiration or censored at date of last observation of follow-up. For cancer-specific survival, all deaths were reviewed and cause of death was determined by the authors (TDC and SBC). Time to diagnosis of successive malignancy was defined as starting from the date of resection for the index lung cancer and ending on the date of diagnosis of successive malignancy.

\section{Statistical Analysis}

Means for parametric continuous data were compared using $t$ tests. Counts and proportions for categoric data were compared using
TABLE 2. Characteristics of successive malignancies diagnosed during follow-up

\begin{tabular}{|c|c|c|c|}
\hline Characteristic & $\begin{array}{c}\text { CT } \\
(n=63)\end{array}$ & $\begin{array}{c}\text { CXR } \\
(n=72)\end{array}$ & $\begin{array}{c}P \\
\text { value }\end{array}$ \\
\hline Type & & & .275 \\
\hline New primary & $14(22 \%)$ & $22(31 \%)$ & \\
\hline Recurrence & $49(78 \%)$ & $50(69 \%)$ & \\
\hline Region & & & .187 \\
\hline Extrathoracic & $16(25 \%)$ & $10(14 \%)$ & \\
\hline Thoracic & $41(65 \%)$ & $51(71 \%)$ & \\
\hline Both & $6(10 \%)$ & $11(15 \%)$ & \\
\hline Location & & & .295 \\
\hline Local & $3(5 \%)$ & $4(6 \%)$ & \\
\hline Regional & $11(17 \%)$ & $14(19 \%)$ & \\
\hline Distant & $34(54 \%)$ & $36(50 \%)$ & \\
\hline Local and regional & $5(8 \%)$ & $1(1 \%)$ & \\
\hline Local and distant & $1(2 \%)$ & 0 & \\
\hline Regional and distant & $9(14 \%)$ & $17(24 \%)$ & \\
\hline Symptomatic at time of detection & $32(51 \%)$ & $58(81 \%)$ & $<.001$ \\
\hline Detected during scheduled surveillance & $46(73 \%)$ & $33(46 \%)$ & .001 \\
\hline Treatment & & & .670 \\
\hline Curative & $26(41 \%)$ & $29(40 \%)$ & \\
\hline Palliative & $29(46 \%)$ & $37(51 \%)$ & \\
\hline Refused & 0 & $1(1 \%)$ & \\
\hline None & $1(1 \%)$ & $1(1 \%)$ & \\
\hline Unknown & $7(11 \%)$ & $4(6 \%)$ & \\
\hline
\end{tabular}

$C T$, Computed tomography; $C X R$, chest x-ray.

chi-square tests. Overall and cancer-specific survival curves were constructed using the Kaplan-Meier method and compared using log-rank tests. Multivariate analysis of prognostic effect of covariates on overall and cancer-specific survival was performed using Cox proportional hazard analysis. To account for potentially confounding covariates and imbalance between the CT and CXR groups with respect to resection procedure and tumor T-stage, propensity score matching using the nearest-neighbor caliper method was carried out to match the CT and CXR groups on the basis of covariates of age, perioperative Charlson comorbidity index, sublobar resection, T-stage of index tumor, and adjuvant therapy with a caliper radius of 0.03 of the logit of the standard deviation of the propensity score. All statistical tests were 2-sided. Analyses were conducted using SPSS version 21.0 (IBM, Armonk, NY). Propensity score matching was performed using the Propensity Score Matching for SPSS plug-in, version 3.0 (Felix Thoemmes, Cornell University, Ithaca, NY).

\section{RESULTS}

\section{Successive Malignancies}

Recurrence or new primary lung cancer was found in $24 \%(135 / 554)$ of patients (Table 2). Overall, successive malignancy was found in $27 \%(63 / 232)$ in the CT group and $22 \%(72 / 322)$ in the CXR group $(P=.195)$. The rate of successive malignancy was $10.7 \%$ per person-year for CT and $6.3 \%$ per person-year for CXR $(P=.002)$. The majority of successive malignancies had thoracic manifestation. The rate of new primary lung cancers overall was $2.1 \%$ per person-year. In the CT group, 9 of 232 patients $(4 \%)$ had false-positive surveillance imaging that 

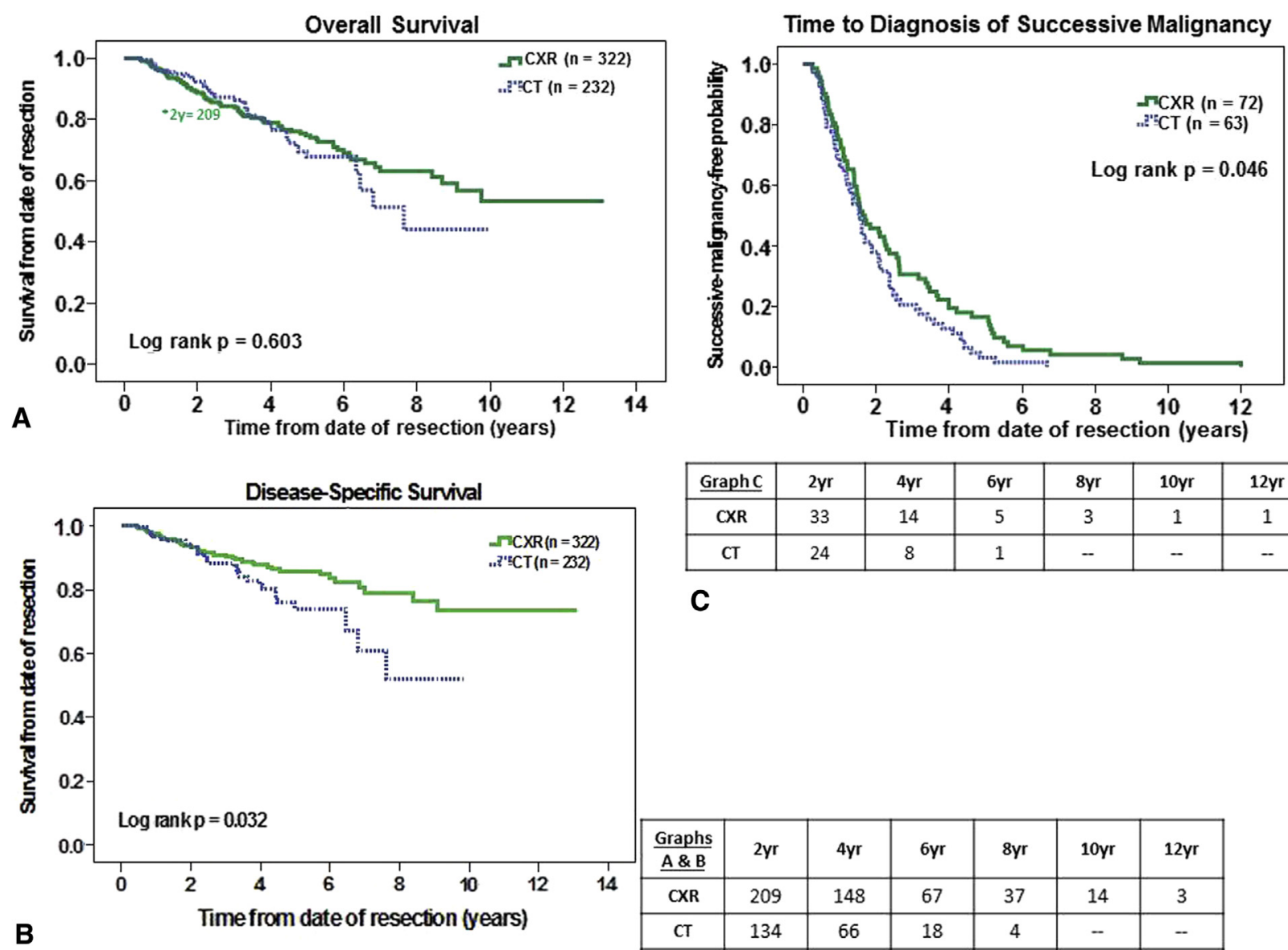

\begin{tabular}{|c|c|c|c|c|c|c|}
\hline GraphC & $2 \mathbf{y r}$ & $4 y \mathbf{r}$ & $6 \mathrm{yr}$ & $8 \mathrm{yr}$ & $10 \mathrm{yr}$ & $12 \mathrm{yr}$ \\
\hline CXR & 33 & 14 & 5 & 3 & 1 & 1 \\
\hline CT & 24 & 8 & 1 & - & - & - \\
\hline
\end{tabular}

C

FIGURE 1. A-C, Kaplan-Meier curves for overall survival, cancer-specific survival, and time to diagnosis of relapse. $C T$, Computed tomography; $C X R$, chest X-ray.

resulted in an invasive diagnostic procedure (bronchoscopy, biopsy, or surgery) versus 1 of 322 patients $(0.3 \%)$ in the CXR group $(P=.002)$. Among patients developing successive malignancy, $49 \%(31 / 63)$ in the CT group were asymptomatic at presentation versus $19 \%(14 / 72)$ in the CXR group $(P<.001)$.

Among patients with successive malignancy, time to diagnosis was shorter for the CT group $(P=.046)$ (Figure 1), with mean times of 1.93 versus 2.56 years for CXR and median times of 1.56 versus 1.63 years for CXR (95\% CI, $1.29-1.83$ vs $1.03-2.23$ ).

\section{Survival}

In unmatched analysis, overall survival was similar between the groups, with 5 -year survival of $67.8 \%$ for CT versus $74.8 \%$ for CXR (mean survival, 7.00 vs 9.19 years; median survival, 7.63 years vs not reached; $P=.603$ ) (Figure 1) (95\% CI, 5.78-9.48). Five-year cancer-specific survival was $74.1 \%$ for CT versus $85.5 \%$ for CXR $(P=.032)$ (Figure 1). Among patients with successive malignancy, there was no difference in overall survival, with 5-year survival of $33.5 \%$ for CT and $40.2 \%$ for
CXR (mean, 4.24 vs 5.34 years; median, 3.96 vs 3.47 years; $P=.843)(95 \%$ CI, 2.71-5.21 vs 2.72-4.22). Among patients with successive malignancy, cancer-specific survival was similar, with 5-year survival of $39.1 \%$ for $\mathrm{CT}$ and $50.7 \%$ for CXR (mean, 4.47 vs 6.51 years; median, 4.44 vs 5.70 years; $P=.353)(95 \% \mathrm{CI}$, 3.33-5.54 vs 3.47-7.93). These results were unchanged if survival was measured from date of diagnosis of successive malignancy.

\section{Multivariate Analysis and Propensity Matching}

In a Cox proportional hazard model, modality of surveillance imaging was not associated with survival $(P=.958)$, and only age, Charlson comorbidity index, and type of resection (sublobar vs nonsublobar resection) were associated with survival (Table 3). Identical results were seen in a model for cancer-specific survival (Table E3).

By using a caliper-based method, 174 propensitymatched pairs were identified (Table E4). There was no significant difference in overall or cancer-specific survival between the groups. Time to diagnosis of successive malignancy trended toward being shorter for CT 
TABLE 3. Regression analysis for overall survival

\begin{tabular}{lccr}
\hline \multicolumn{1}{c}{ Covariate } & $\begin{array}{c}\text { Hazard } \\
\text { ratio }\end{array}$ & $\mathbf{9 5 \% \text { CI }}$ & $\begin{array}{r}\boldsymbol{P} \\
\text { value }\end{array}$ \\
\hline Imaging (CXR vs CT) & 0.988 & $0.629-1.551$ & .958 \\
Age & 1.051 & $1.029-1.073$ & $<.001$ \\
Charlson index & 1.359 & $1.151-1.605$ & $<.001$ \\
Resection (sublobar vs nonsublobar) & 2.577 & $1.614-4.115$ & $<.001$ \\
Tumor T-stage (1 vs 2a) & 0.862 & $0.510-1.456$ & .578 \\
Tumor size & 1.108 & $0.876-1.401$ & .393 \\
Histology (non-BAC vs BAC) & 1.581 & $0.812-3.077$ & .178 \\
Adjuvant therapy (absent vs present) & 0.691 & $0.389-1.226$ & .206 \\
Gender (male vs female) & 1.265 & $0.864-1.852$ & .227 \\
Race (non-Caucasian vs Caucasian) & 1.085 & $0.566-2.080$ & .806 \\
\hline BAC, B
\end{tabular}

$B A C$, Bronchioloalveolar carcinoma; $C I$, confidence interval; $C T$, computed tomography; $C X R$, chest x-ray.

(median, 1.60 vs 2.10 years for CXR) (95\% CI, 1.23-1.96 vs $0.87-3.34 ; P=.058$ ) (Figure 2 ).

There was no difference between groups in the proportion of successive malignancies that were treated with curative intent, accounting for $41 \%(26 / 63)$ in the CT group versus $40 \%$ (29/72) in the CXR group $(P=.907)$ (Figure E3). Overall and cancer-specific survivals were similar between groups when successive malignancy was treated with curative intent $(P=.369)$ or when patients received palliative therapy $(P=.655)$ (Figure E3). Overall and cancer-specific survivals were also similar between groups among patients developing new primary lung cancers.

In both groups, those given palliative treatment had shorter survival compared with patients given curativeintent treatment, with a median difference of 52 months (median survival, 2.64 vs 6.98 years; $P<.001$ ). Patients whose successive malignancy was detected during a scheduled follow-up visit had similar overall and cancerspecific survival versus those who presented outside of a scheduled follow-up. The difference between overall survival of patients with asymptomatic versus symptomatic presentation of successive malignancy trended toward statistical significance (mean, 5.41 vs 4.78 years; median, 4.98 vs 3.23 years; $P=.070)(95 \% \mathrm{CI}, 3.30-6.66$ vs 2.45-4.00). However, asymptomatic patients were more likely to be offered curative-intent treatment compared with those presenting symptomatically $(56 \%$ [25/45] vs $33 \%[30 / 90] ; P=.013)$ and to have longer cancer-specific
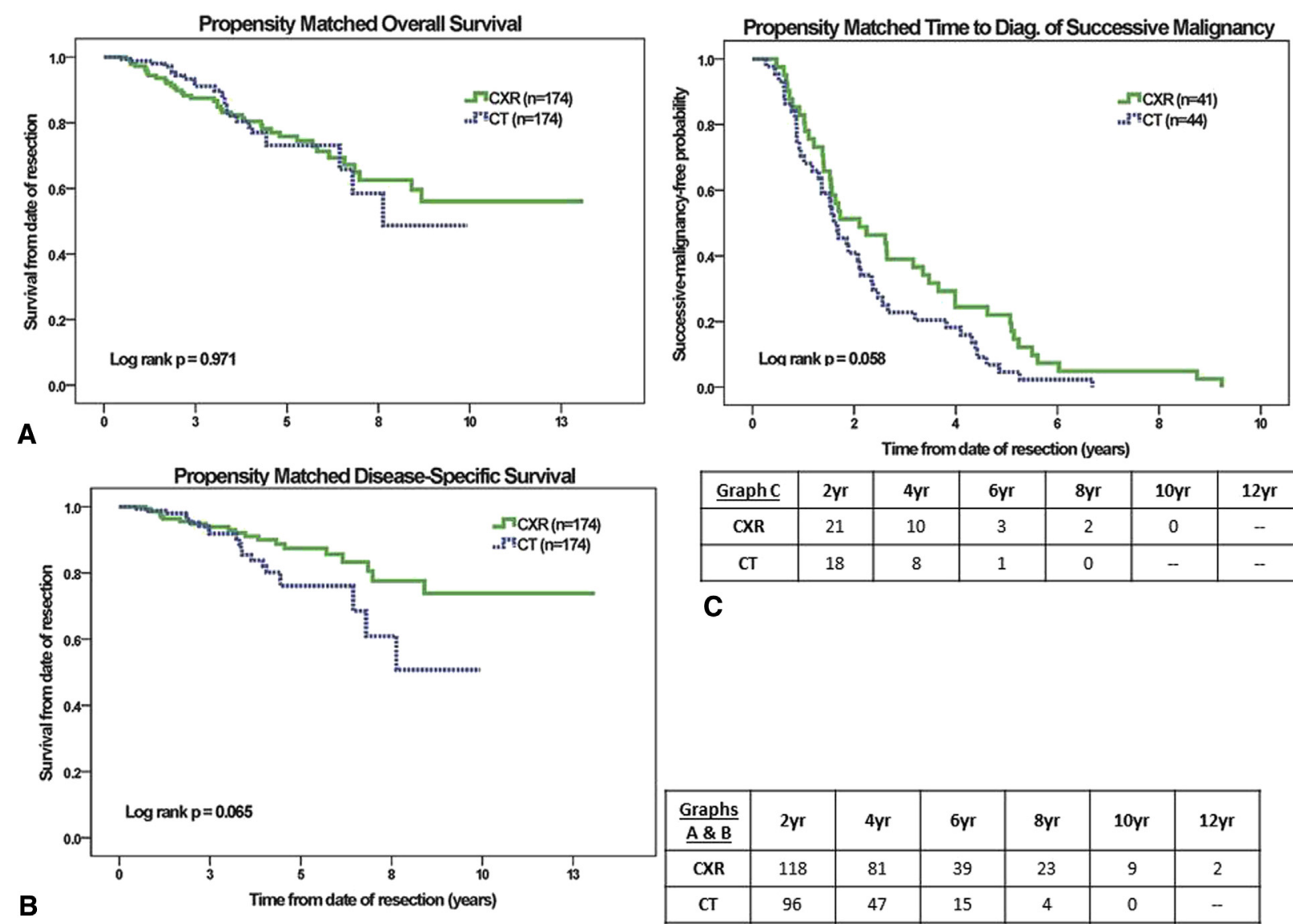

\begin{tabular}{|c|c|c|c|c|c|c|}
\hline GraphC & $2 \mathbf{y r}$ & $4 \mathbf{y r}$ & $\mathbf{6 y r}$ & $\mathbf{8 y r}$ & $\mathbf{1 0 y r}$ & $\mathbf{1 2 y r}$ \\
\hline CXR & 21 & 10 & 3 & 2 & 0 & - \\
\hline CT & 18 & 8 & 1 & 0 & - & - \\
\hline
\end{tabular}

\section{C}

\begin{tabular}{|c|c|c|c|c|c|c|}
\hline$\frac{\text { Graphs }}{\mathbf{A} \& \mathbf{B}}$ & $2 \mathbf{y r}$ & $4 \mathbf{y r}$ & $6 \mathbf{6 y r}$ & $8 \mathrm{yr}$ & $\mathbf{1 0 y r}$ & $12 \mathrm{yr}$ \\
\hline CXR & 118 & 81 & 39 & 23 & 9 & 2 \\
\hline CT & 96 & 47 & 15 & 4 & 0 & - \\
\hline
\end{tabular}

FIGURE 2. A-C, Kaplan-Meier curves for propensity-matched overall survival, cancer-specific survival, and time to diagnosis of relapse. $C T$, Computed tomography; $C X R$, chest x-ray. 
survival (mean, 6.32 vs 5.45 years; median, 6.15 vs 3.47 years; $P=.019)(95 \% \mathrm{CI}, 4.88-7.42$ vs $2.67-4.27)$.

\section{DISCUSSION}

Given significant variability in the risk of recurrence and treatment strategies for early-stage versus locally advanced NSCLC, we chose to examine the stage I population alone. These data demonstrate that for pathologic stage I NSCLC, surveillance with CT imaging was associated with earlier detection of successive malignancy compared with CXR imaging in the unmatched comparison with a similar trend in the propensity-matched comparison. Patients with successive malignancy who underwent CT were more likely to be diagnosed at an asymptomatic stage or at a scheduled follow-up visit than those who underwent CXR. Patients with asymptomatic successive malignancies were significantly more likely to receive curative-intent treatment compared with those presenting symptomatically. However, there was no demonstrable improvement in overall survival with $\mathrm{CT}$ surveillance versus CXR. Furthermore, despite the association between $\mathrm{CT}$ and the detection of asymptomatic successive malignancies, CT did not result in a greater likelihood of patients receiving curative-intent therapy. Thus, earlier identification of lesions by $\mathrm{CT}$ and identification of asymptomatic lesions did not result in a survival benefit in our cohort.

The appropriate modality and regimen for postoperative radiographic surveillance of patients after surgical resection for NSCLC remain unresolved. Although findings from the NLST trial indicate improved mortality with CT screening for a first primary lung cancer among high-risk smokers, ${ }^{10}$ it is unproven whether CT surveillance after resection for lung cancer improves mortality from a second lung cancer (Table E2). A primary goal of postoperative surveillance after lung resection is the early detection of new primary lung cancers, which have been shown to occur at a rate of $1 \%$ to $6 \%$ per person-year (with most studies reporting $1 \%-2 \%$ per person-year). ${ }^{5-9}$ Intuitively, the risk of developing a new primary lung cancer would seem higher among patients who have undergone resection for lung cancer compared with high-risk smokers without a history of lung cancer as represented in the NLST. The risk of new primary lung cancer is additive to the risk of recurrence in previously resected patients, and our data reinforce the lack of clarity in whether intensive surveillance improves survival of patients with recurrence, even with earlier detection.

Various studies have similarly examined the effectiveness of surveillance CT imaging of patients after resection for lung cancer. A widely cited rationale for the use of CT imaging in surveillance of patients with early-stage lung cancer is to detect new primary lung cancers early. ${ }^{5,6,11}$ In a study of patients in stage I and II undergoing CT surveillance after resection, ${ }^{5}$ scheduled $\mathrm{CT}$ scans detected a majority of successive malignancies, consistent with our observation. Most series have demonstrated that the rate of new primary lung cancer development after cancer resection is $1 \%$ to $2 \%$ per person-year. ${ }^{6-9}$ Likewise, we observed a rate of new primary lung cancers of $2.1 \%$ per person-year for our patients with pathologic stage I NSCLC. Others have observed that the majority of second primary lung cancers were detected at stage I and treated by surgical resection. ${ }^{5}$ We demonstrated that new primary lung cancers were associated with significantly longer survival than recurrences. Furthermore, among patients diagnosed with new primaries, CT was not associated with significantly longer survival. Thus, it remains unclear whether surveillance $\mathrm{CT}$ imaging enhances the survival of patients who develop new primary lung cancers.

Hanna and colleagues ${ }^{14}$ studied a population with predominantly early-stage NSCLC (79\% stage I) who underwent surveillance with simultaneous CXR and minimal-dose CT scans and found that minimal-dose CT resulted in improved sensitivity and negative predictive value versus CXR in the detection of successive malignancies. They demonstrated that patients whose successive malignancies were detected asymptomatically had a markedly greater rate of curative-intent treatment compared with symptomatic patients and that those treated with curative intent had improved survival compared with those treated palliatively. We did not observe a significant difference in the rate of curative-intent treatment for the CT and CXR groups, even though CT surveillance was more likely to detect successive malignancies asymptomatically. The minimal-dose CT evaluated by Hanna and colleagues $^{14}$ results in less radiation exposure $(0.2 \mathrm{mSv})$ but may be limited in the ability to delineate early subsolid adenocarcinomas or mediastinal recurrence. Our observations suggest that the efficacy of CT imaging in detecting successive malignancies asymptomatically may not be a sufficiently reliable surrogate marker for assessing impact on treatment or survival.

The results of our study are consistent with those from earlier studies that have directly compared CT with radiograph follow-up regimens. Nakamura and colleagues ${ }^{17}$ reported similar survival for patients in stage I by using surveillance with CT and CXR versus CXR alone. Benamore and colleagues ${ }^{15}$ found that, for patients with stage IIB-III, follow-up with routine CT offered earlier detection of successive malignancy but did not result in improved survival versus CXR. Virgo and colleagues, ${ }^{18}$ comparing nonintensive and intensive follow-up that included more frequent CT imaging, found that intensive follow-up did not improve survival. Likewise, Younes and colleagues ${ }^{20}$ observed that routine follow-up with CT and CXR did not improve survival compared with symptomprompted follow-up. 
Any survival advantage with CT surveillance must be considered in the context of cost-effectiveness and potential hazards of false-positive CT imaging that may result in unnecessary cost and patient morbidity. Postoperative CT imaging has been associated with a nontrivial falsepositive rate of $50 \%$ for patients with stage I-IV disease. ${ }^{21}$ Hanna and colleagues ${ }^{14}$ found that the positive predictive value of minimal-dose CT was significantly lower than that of CXR for surveillance of patients with stage I-IV $(25.1 \%$ vs $91.7 \%)$. Furthermore, reports have demonstrated that $4 \%$ to $5 \%$ of patients undergoing CT surveillance underwent invasive diagnostic procedures for false-positive imaging. ${ }^{5,21}$ The rate of false-positives necessitating invasive diagnostic procedures of $4 \%$ in our CT group was similar and was higher than in the CXR group. Therefore, the use of intensive CT screening carries a small but meaningful risk of unnecessary invasive procedures.

We certainly recognize the significant limitations of our study in its retrospective approach and the potential lack of power based on our cohort size to detect an actual survival difference. Because we sought to identify and compare patients who consistently received CXR or CT surveillance imaging, we had to exclude patients whose surveillance protocol was inconsistent in the imaging modality used for routine surveillance. The lack of difference in the rate of curative intent therapy may be related to a nonsignificant increase in the number of new primary cancers diagnosed in the CXR group versus the $\mathrm{CT}$ group given that the length of follow-up was longer for the CXR group, allowing for more time to diagnose new primary lesions. Our simple power calculation indicated that with a power of $80 \%$ and an alpha level of $5 \%$, our sample size was sufficient to detect a $29 \%$ difference in median survival; differences less than $29 \%$ could not be detected. Given the limitations of our sample size, we have included sample size calculations for theoretic scenarios of surveillance trials in Table E5. It was also not possible to assess whether the quality of life of patients was enhanced or diminished by earlier diagnosis of successive malignancies in the CT group. The ongoing IFCT-0302 trial, ${ }^{22}$ comparing follow-up using CXR versus CT and bronchoscopy, will help determine the survival benefits of intensive follow-up after resection.

\section{CONCLUSIONS}

Consistent with our primary hypothesis, our study suggests that for patients with stage I lung cancer treated by surgical resection, routine surveillance CT was associated with earlier diagnosis of recurrence or new primary lung cancer compared with routine CXR. However, this earlier diagnosis did not result in a demonstrable difference in treatment approach or overall survival. By recognizing the limitations of our study but also acknowledging the consistency of our findings with the findings of others, we conclude that the appropriate imaging modality and regimen for surveillance remain unclear and unsubstantiated and recommend that a randomized controlled, multi-institutional prospective clinical trial be performed to help define the appropriate radiographic modality for surveillance after resection for lung cancer.

\section{References}

1. Feld R, Rubinstein LV, Weisenberger TH, and the Lung Cancer Study Group. Sites of recurrence in resected stage I non-small-cell lung cancer: a guide for future studies. J Clin Oncol. 1984;2:1352-8.

2. Goodgame B, Viswanathan A, Zoole J, Gao F, Miller CR, Subramanian J, et al. Risk of recurrence of resected stage I non-small cell lung cancer in elderly patients as compared with younger patients. J Thorac Oncol. 2009; 4:1370-4.

3. Martini N, Melamed MR. Multiple primary lung cancers. J Thorac Cardiovasc Surg. 1975;70:606-12.

4. Walsh GL, O'Connor M, Willis KM, Milas M, Wong RS, Nesbitt JC, et al. Is follow-up of lung cancer patients after resection medically indicated and cost-effective? Ann Thorac Surg. 1995;60:1563-72.

5. Lou F, Huang J, Sima CS, Dycoco J, Rusch V, Bach PB. Patterns of recurrence and second primary lung cancer in early-stage lung cancer survivors followed with routine computed tomography surveillance. J Thorac Cardiovasc Surg. 2013; 145:75-82

6. Lamont JP, Kakuda JT, Smith D, Wagman LD, Grannis FW. Systematic postoperative radiologic follow-up in patients with non-small cell lung cancer for detecting second primary lung cancer in stage IA. Arch Surg. 2002;137: 935-9.

7. Senthi S, Lagerwaard FJ, Haasbeek CJA, Slotman BJ, Senan S. Pattern of disease recurrence after stereotactic ablative radiotherapy for early stage non-small-cell lung cancer: a retrospective analysis. Lancet Oncol. 2012;13:802-9.

8. Johnson BE. Second lung cancers in patients after treatment for an initial lung cancer. J Natl Cancer Inst. 1998;90:1335-45.

9. Colt HG, Murgu SD, Korst RJ, Slatore CG, Unger M, Quadrelli S. Follow-up and surveillance of the patient with lung cancer after curative-intent therapy. Chest 2013; 143:e437S-54S.

10. The National Lung Screening Trial Research Team. Reduced lung-cance mortality with low-dose computed tomographic screening. N Engl J Med. 2011;365:395-409.

11. Jaklitsch MT, Jacobson FL, Austin JHM, Field JK, Jett JR, Keshavjee S, et al The American Association for Thoracic Surgery guidelines for lung cancer screening using low-dose computed tomography scans for lung cancer survivors and other high-risk groups. J Thorac Cardiovasc Surg. 2012;144:33-8.

12. Crinò L, Weder W, van Meerbeeck J, Felip E. Early stage and locally advanced (non-metastatic) non-small-cell lung cancer: ESMO Clinical Practice Guidelines for diagnosis, treatment and follow-up. Ann Oncol. 2010; 21:v103-15.

13. Ettinger DS, Akerley W, Borghaei H, Chang AC, Cheney RT, Chirieac LR, et al Non-small cell lung cancer. J Natl Compr Canc Netw. 2012;10:1236-71.

14. Hanna WC, Paul NS, Darling GE, Moshonov H, Allison F, Waddell TK, et al Minimal-dose computed tomography is superior to chest x-ray for the followup and treatment of patients with resected lung cancer. J Thorac Cardiovasc Surg. 2014;147:30-5

15. Benamore R, Shepherd FA, Leighl N, Pintilie M, Patel M, Feld R, et al. Does intensive follow-up alter outcome in patients with advanced lung cancer? J Thorac Oncol. 2007;2:273-81.

16. Chiu CH, Chern MS, Wu MH, Hsu WH, Wu YC, Huang MH, et al. Usefulness of low-dose spiral CT of the chest in regular follow-up of postoperative non-small cell lung cancer patients: preliminary report. J Thorac Cardiovasc Surg. 2003; 125:1300-5.

17. Nakamura R, Kurishima K, Kobayashi N, Ishikawa S, Goto Y, Sakai M, et al. Postoperative follow-up for patients with non-small cell lung cancer. Onkologie. 2010;33:14-8.

18. Virgo KS, McKirgan LW, Caputo MC, Mahurin DM, Chao LC, Caputo NA, et al Post-treatment management options for patients with lung cancer. Ann Surg. 1995;222:700-10. 
19. Westeel V, Choma D, Clément F, Woronoff-Lemsi MC, Pugin JF, Dubiez A, et al. Relevance of an intensive postoperative follow-up after surgery for non-small cell lung cancer. Ann Thorac Surg. 2000;70:1185-90.

20. Younes RN, Gross JL, Deheinzelin D. Follow-up in lung cancer: how often and for what purpose? Chest. 1999;115:1494-9.

21. Korst RJ, Kansler AL, Port JL, Lee PC, Altorki NK. Accuracy of surveillance computed tomography in detecting recurrent or new primary lung cancer in patients with completely resected lung cancer. Ann Thorac Surg. 2006;82: 1009-15.

22. Westeel V, Lebitasy MP, Mercier M, Girard P, Barlesi F, Blanchon F, et al. IFCT-0302 trial: randomised study comparing two follow-up schedules in completely resected non-small cell lung cancer [in French]. Rev Mal Respir. 2007;24:645-52.

\section{Discussion}

Dr Robert Korst (Paramus, $N J$ ). The question of how to follow patients with lung cancer after potentially curative treatment remains unresolved but has recently begun to focus on the role of CT scanning in this capacity. This focus has been potentiated by the findings of the NLST, which demonstrated that screening CT saves lives in individuals who meet high-risk criteria.

Whether or not to use CT to follow patients after curative resection is a 2-part question: First, is it effective in detecting metachronous lung cancer, and, second, even if it is effective, does it enhance survival? Dr Crabtree and colleagues should be commended because they are attempting to address both of these questions in their study. Although an emerging body of literature has suggested that $\mathrm{CT}$ is a sensitive test for the detection of recurrent and new primary lung cancer in the surveillance setting, few studies have addressed the survival part of the question in detail.

Although the study is retrospective and perhaps somewhat underpowered, it is well carried out and the data are complete. In addition, when it was discovered that the CT and CXR groups were not identical with regard to tumor stage and type of resection, the authors used propensity score matching and a multivariate analysis to try and compensate.

I have 3 questions regarding the study. My first question has to do with the lack of a difference in survival between the 2 groups. This may be because there was no difference in the percent of patients offered curative-intent treatment between the 2 groups. It is intuitive that patients who are followed with CT will have their secondary disease picked up sooner, with more patients being asymptomatic, thereby allowing more attempts at curative resection or curative treatment. Although the first 2 of these were indeed demonstrated in this study, it didn't translate into more patients undergoing curative treatments. Why do you think this is? Could it be because the patients in the CXR group were more likely to have second primary cancers discovered or at least equally as likely to have second primary cancers discovered? I know it didn't reach statistical significance. Given that CT is more sensitive than CXR for detecting pulmonary nodules, how do you explain the fact that a greater proportion of patients, or at least as many patients, had second primary lung cancers in the CXR group compared with the CT group?

Dr Crabtree. Specifically to address that last question, we did attempt to look at the new primary lung cancers that were diagnosed as a subset, and the numbers get smaller, so it becomes harder to analyze. Patients in the CXR cohort were accrued earlier in the study with a median length of follow-up of 3.5 years versus 2.5 years for the CT group. There was a nonsignificant difference in the overall rate of new primary lesions diagnosed, with more in the CXR group. Also, the median time to diagnosis of new primary lesions was longer in the CXR group versus CT ( 3.7 vs 2.1 years). It is possible that the longer follow-up for the CXR group resulted in the identification of more new primary lesions. This may have contributed to the similar rates of curative intent treatment between the groups. Again, however, it could be a sample size issue. There were only 14 new primary lesions in the CT group and 22 in the CXR group.

Dr Korst. It would be intuitive that more patients would be detected with second primary lung cancers in the CT group. Another potential explanation for the lack of a difference in curative-intent treatments between the 2 groups could be that the CT group could have been less physically fit or had worse pulmonary function than the CXR group. I know you had a comorbidity index in there. This is kind of implied, because in the CT group, patients were 2.5 times more likely to have a limited resection at the time of their initial operation than the CXR group. Did you look at that? Do you know if that was the case? There was a profound difference in the groups between the types of operations done at the first time around, and perhaps those patients, their pulmonary functions were poorer. Maybe that is why they didn't undergo curative-intent treatments the second time around.

Dr Crabtree. That is a great point. I don't have an objective answer for you on that. The higher proportion of limited resections may have contributed to this difference in the unmatched comparison; however, our propensity-matched analysis took into account the disparity in the proportion of limited resections, which ultimately demonstrated no difference in survival. Our comorbidity index was similar between the groups and was also used in the propensity matching model, so I can't objectively say that the patients in the CT cohort were sicker.

Dr Korst. Sure. Although I agree with the overall conclusion of the article, which is obviously that a formal randomized, controlled trial should be performed; this will obviously take years to accomplish. On the basis of your results, and I know you have a lot of surgeons in your practice, is anyone going to change anything?

Dr Crabtree. We have over the years transitioned to CT, again, for the same reason, that we instinctively think that CT should be better, although we haven't been able to prove it. I think we have anecdotally changed the frequency of imaging. Rather than doing a CT scan every 3 months, we have consistently tried to go to every 6 months for 2 years, and then once a year. I think looking to the future, though, we may be thinking one-sided about this. We are trying to say one size fits all for all patients, but it may be in a trial that we determine that there is a way to customize it according to the tumor, perhaps a genomic signature or lymphovascular invasion. Maybe there are multiple factors that not only affect how we treat those patients but also how we follow them in terms of the frequency and the imaging modality.

Dr Harvey Pass (New York, NY). You measured the time to death from the time of the detection of the recurrence; is that right?

Dr Crabtree. We measured the time of death starting from the time of resection at the original operation, and the goal of that is to potentially eliminate or limit the lead-time bias that you would see in some of the screening trials. 
Dr Pass. But if you are going to give potentially curative treatment and the patient has recurrence, then you want to see how long that patient is going to live and if he/she is going to die quicker because of later disease, as with symptomatic patients. That may be what you really want to find.

Dr Crabtree. So you're asking me if we went from the time of diagnosis of the recurrence?

Dr Pass. Yes, exactly, how long they live from the time of the diagnosis in each group.

Dr Crabtree. We did a separate analysis on that, which I didn't present, and the survival from the time of diagnosis of recurrence was still similar. Again, it could be a sample size issue.

Dr Pass. The biology of a second primary is a lot different than patients with local or systemic recurrence. You say you broke down the data with regard to CT and CXR for second primaries. Did you do that for patients who had a locoregional or systemic recurrence only?

Dr Crabtree. The truth is, as the Toronto group presented last year, I don't think it matters whether we call it a recurrence or a new primary, as long as the goal of treatment was curative. We did look at recurrences separately, however. The time to diagnosis of recurrences was similar between the cohorts (1.3 vs 1.4 years) and shorter compared with the time to diagnosis of new primary lesions. Also, overall survival was similar for recurrences, although we did not break them down further into systemic versus locoregional.

Dr Joshua Sonett (New York, NY). Given the number of patients who were needed for the NLST, I was impressed that you picked up any difference with the asymptomatic and the earlier diagnosis with such a small cohort. Given that, I wouldn't expect you to find a survival difference with such a small cohort.
I have 2 questions. If you are designing a prospective study, how many patients do you think you would need? Think of the large volume with the NLST. Two, given the results of the NLST, which put these patients basically in the high-risk group where we think they are going to have a survival advantage with a CT scan every year, how would you randomize them to not getting a CT scan?

Dr Crabtree. To answer your first question, I think one of the reasons we were able to detect a difference is because we had such a long accrual time and a follow-up time. For a randomized trial, I did a couple of models to look at that. If we use the International Association for the Study of Lung Cancer data, if we looked at specifically IA lung cancers and did a 3-year accrual and 5 years of follow-up, with a $90 \%$ power to detect a $20 \%$ difference, with approximately $10 \%$ attrition rate, we would need approximately 2000 patients in each group. If we specifically did the same parameters for just IIIA disease, then we would need approximately 700 patients in each group with that follow-up time. What was the second question, Dr Sonett?

Dr Sonett. How do you reconcile just getting CXRs when these patients are in the high-risk group for the NLST for surveillance?

Dr Crabtree. This was a retrospective study.

Dr Sonett. No, if you did a prospective study.

Dr Crabtree. If we did, I think we would have a discussion and would likely be looking at examining different types of CT scan, whether it be low-dose or contrast CT or even, again, the consideration of PET-CT for more advanced-stage disease. I suspect it would be hard to find enough surgeons who would agree on performing a CXR in a randomized study.

\title{
EDITORIAL COMMENTARY
}

\section{Lung cancer survivors need lung cancer screening}

\author{
Raja M. Flores, MD
}

\section{See related article on pages 45-53.}

Surveillance of patients with lung cancer after curative resection is essentially just screening for lung cancer. The success of screening relies on identifying early disease

\footnotetext{
From the Department of Thoracic Surgery, Mount Sinai Health System, Icahn School of Medicine at Mount Sinai, New York, NY.

Disclosures: Author has nothing to disclose with regard to commercial support.

Received for publication Sept 16, 2014; accepted for publication Sept 27, 2014; available ahead of print Oct 22, 2014.

Address for reprints: Raja M. Flores, MD, Department of Thoracic Surgery, Mount Sinai Health System, One Gustave L. Levy Place, Box 1023, New York, NY 10029 (E-mail: raja.flores@mountsinai.org).

J Thorac Cardiovasc Surg 2015;149:53-4

$0022-5223 / \$ 36.00$

Copyright (c) 2015 by The American Association for Thoracic Surgery

http://dx.doi.org/10.1016/j.jtcvs.2014.09.115
}

and implementing curative surgery. A critical element of successful screening is the proper identification of a highrisk group. What better group than those who have already had lung cancer? A computed tomography (CT) screening program evaluating 20 year-old nonsmokers for lung cancer would clearly result in failure because the incidence of disease in this patient population is close to zero. The National Lung Screening Trial (NLST) clearly identified a group of smokers at a high enough risk of developing lung cancer to demonstrate a mortality reduction when $\mathrm{CT}$ screening was followed by early surgical intervention. ${ }^{1}$ The trial included 33 different sites across the nation and cost more than US\$250 million to conduct. The outcome of the trial proved that treatment of smaller, earlier stage tumors resulted in a better cure rate than larger, later stage tumors. We do not need to spend resources on another randomized 


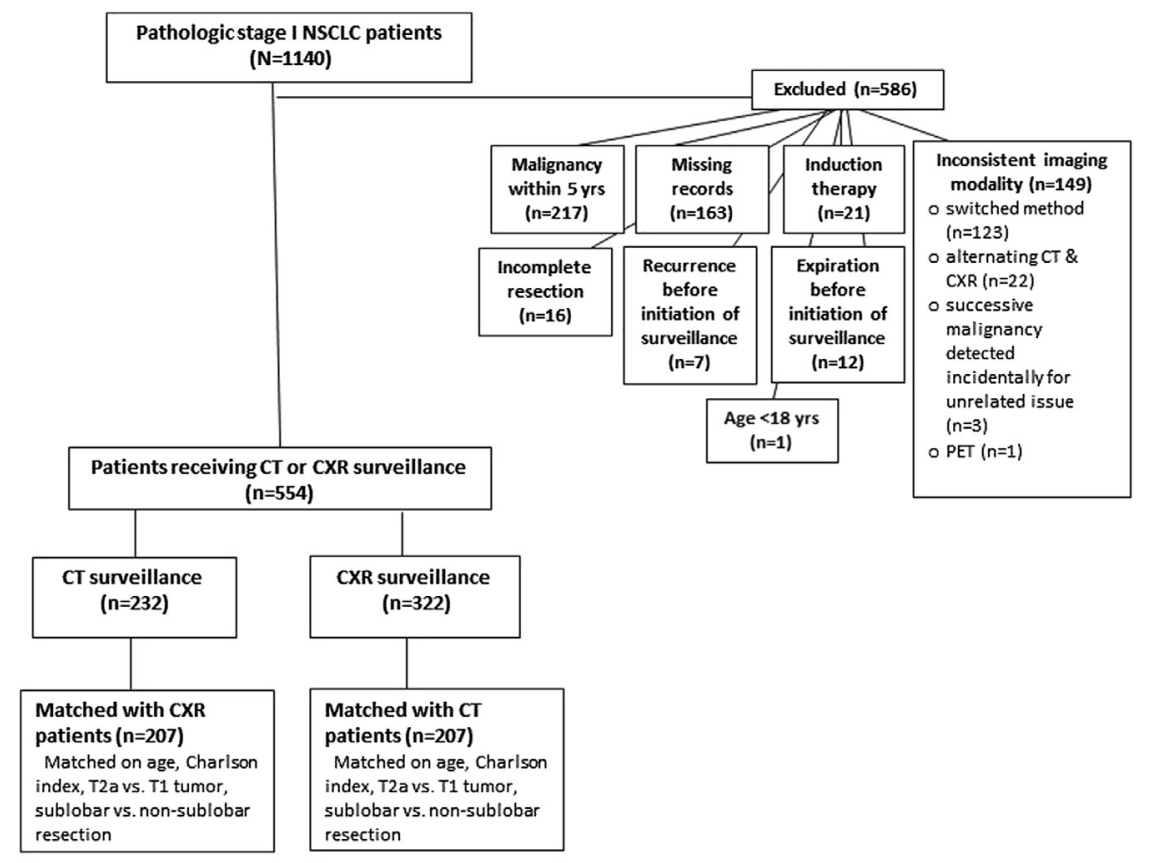

FIGURE E1. Overall CONSORT diagram for surveillance imaging after resection for pathologic stage I lung cancer. $C T$, Computed tomography; $C X R$, chest x-ray; NSCLC, non-small cell lung cancer; PET, positron emission tomography.

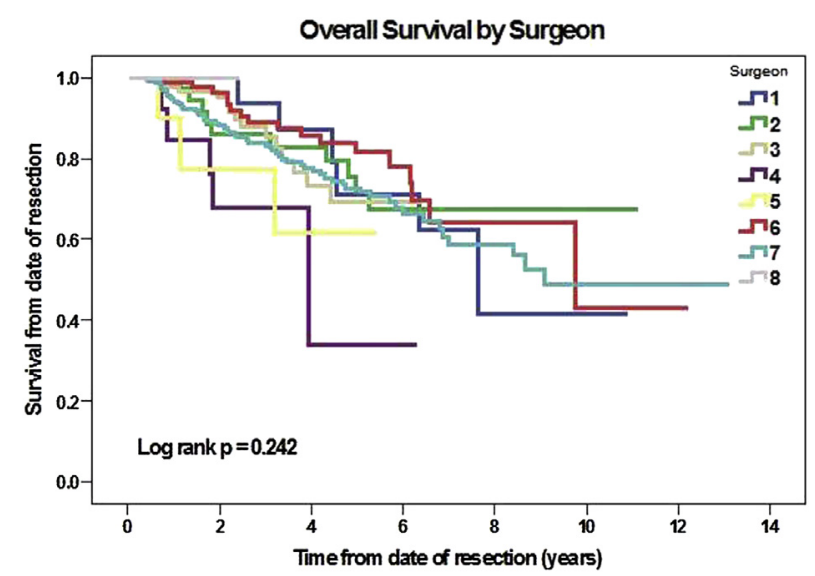

FIGURE E2. Kaplan-Meier curves for overall survival based on treating surgeon.

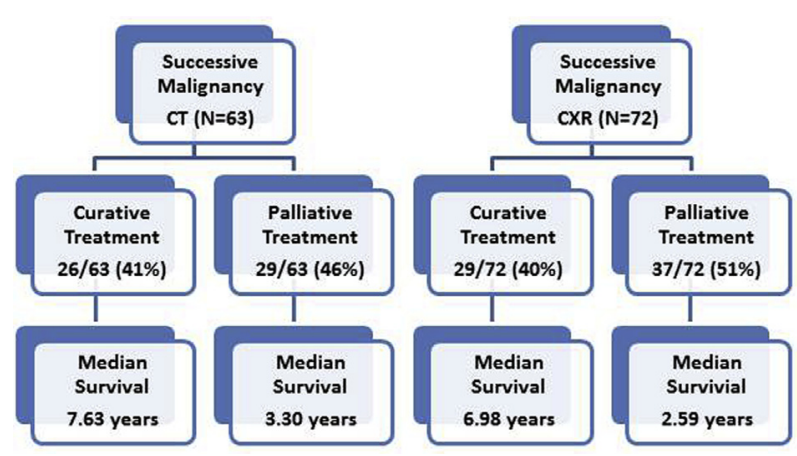

FIGURE E3. CONSORT diagram of diagnosis, treatment, and survival of successive malignancy using CT or CXR for postoperative surveillance. There are 8 patients in CT group and 6 patients in CXR group who had no treatment, who refused, or who had unknown treatment status. $C T$, Computed tomography; $C X R$, chest x-ray. 
TABLE E1. Recent guidelines for radiographic surveillance after resection for non-small cell lung cancer ${ }^{9,11-13}$

\begin{tabular}{ll}
\hline \multicolumn{1}{c}{ Agency (y) } & \multicolumn{1}{c}{ Recommendations } \\
\hline American Association for Thoracic Surgery $(2012)^{11}$ & $\begin{array}{c}\text { High-resolution CT for first } 4 \text { y (with baseline CT at } 6 \text { mo and scans at least every } 6 \text { mo } \\
\text { for first } 3 \text { y), then annual low-density CT annually thereafter } \\
\text { Chest CT every } 6 \text { mo for } 2 \text { y, annually thereafter }\end{array}$ \\
American College of Chest Physicians $(2013)^{9}$ & CT every 6 mo for 2 y, annually thereafter \\
European Society of Medical Oncology $(2010)^{12}$ & Helical chest CT with or without contrast every 6-12 mo for 2 y, chest CT without \\
National Comprehensive Cancer Network $(2012)^{13}$ & contrast annually thereafter
\end{tabular}

$C T$, Computed tomography.

TABLE E2. Findings from surveillance studies of non-small cell lung cancer survivors ${ }^{4-6,14-20}$

\begin{tabular}{|c|c|c|}
\hline Reference & Patients and comparison & Findings \\
\hline $\begin{array}{l}\text { Benamore and colleagues } \\
\qquad(\text { Canada, 2007) })^{15}\end{array}$ & $\begin{array}{l}\mathrm{n}=75, \text { stage IIB-III, routine CXR and CT vs } \\
\text { routine CXR with CT done on clinical suspicion }\end{array}$ & No significant difference in disease-free or overall survival \\
\hline $\begin{array}{l}\text { Chiu and colleagues } \\
\left(\text { Taiwan, 2003) }^{16}\right.\end{array}$ & $\begin{array}{l}\mathrm{n}=73, \text { stage I-IV, follow-up with concurrent } \\
\text { CXR and LDCT }\end{array}$ & $\begin{array}{l}\text { Majority of relapses were detected by LDCT. LDCT more sensitive } \\
\text { than CXR. }\end{array}$ \\
\hline $\begin{array}{l}\text { Hanna and colleagues } \\
\qquad(\text { Canada, 2014) }\end{array}$ & $\begin{array}{l}\mathrm{n}=271, \text { stage I-IV, follow-up with concurrent } \\
\text { CXR and MnDCT }\end{array}$ & $\begin{array}{l}\text { Majority of relapses were detected asymptomatically. MnDCT more } \\
\text { sensitive than CXR. Asymptomatic relapses had greater rate of } \\
\text { curative treatment and longer survival. }\end{array}$ \\
\hline $\begin{array}{l}\text { Lamont and colleagues } \\
\quad(\text { USA, 2002) }\end{array}$ & $\mathrm{n}=124$, stage I-III, follow-up with CT and CXR & Majority of relapses were detected by follow-up CT. \\
\hline $\begin{array}{l}\text { Lou and colleagues } \\
(\text { USA, 2013) }\end{array}$ & $\mathrm{n}=1294$, stage I-II, follow-up with CT & Majority of relapses were detected by follow-up CT. \\
\hline $\begin{array}{l}\text { Nakamura and colleagues } \\
\quad(\text { Japan, 2010) }\end{array}$ & $\begin{array}{r}\mathrm{n}=1389 \text {, stage I-IIIB, follow-up by pulmonologists } \\
\text { with CXR and CT vs thoracic surgeons with CXR }\end{array}$ & $\begin{array}{l}\text { No significant difference in survival for stage I, CXR/CT group had } \\
\text { longer survival for stage II-III. }\end{array}$ \\
\hline $\begin{array}{l}\text { Virgo and colleagues } \\
(\text { USA, 1995) }\end{array}$ & $\begin{array}{l}\mathrm{n}=182, \text { stage I-IIIA, intensive vs nonintensive } \\
\text { follow-up }\end{array}$ & No significant difference in survival. \\
\hline $\begin{array}{l}\text { Walsh and colleagues } \\
\text { (USA, 1995) }\end{array}$ & $\mathrm{n}=358$, stage I-IIIB & $\begin{array}{l}\text { Asymptomatically detected relapses not associated with longer } \\
\text { survival. }\end{array}$ \\
\hline $\begin{array}{l}\text { Westeel and colleagues } \\
(\text { France, 2000) })^{19}\end{array}$ & $\begin{array}{l}\mathrm{n}=192, \text { stage I-IV, follow-up with CXR, CT, } \\
\text { and bronchoscopy }\end{array}$ & Asymptomatically detected relapses associated with longer survival. \\
\hline $\begin{array}{l}\text { Younes and colleagues } \\
(\text { Brazil, 1999) }\end{array}$ & $\begin{array}{l}\mathrm{n}=130, \text { stage I-IIIB, routine CXR and CT vs } \\
\text { symptom-prompted follow-up }\end{array}$ & No significant difference in disease-free or overall survival. \\
\hline
\end{tabular}

TABLE E3. Regression analysis for cancer-specific survival

\begin{tabular}{lccc}
\hline \multicolumn{1}{c}{ Covariate } & $\begin{array}{c}\text { Hazard } \\
\text { ratio }\end{array}$ & $\mathbf{9 5 \%}$ CI & $\begin{array}{c}\boldsymbol{P} \\
\text { value }\end{array}$ \\
\hline Imaging (CXR vs CT) & 0.686 & $0.396-1.187$ & .178 \\
Age & 1.041 & $1.015-1.069$ & .002 \\
Charlson index & 1.282 & $1.030-1.595$ & .026 \\
Resection (sublobar vs nonsublobar) & 1.893 & $1.040-3.443$ & .037 \\
Tumor T-stage (1 vs 2a) & 0.719 & $0.379-1.361$ & .311 \\
Tumor size & 1.032 & $0.774-1.375$ & .831 \\
Histology (non-BAC vs BAC) & 1.863 & $0.738-4.702$ & .188 \\
Adjuvant therapy (absent vs present) & 0.633 & $0.321-1.246$ & .186 \\
Gender (male vs female) & 1.078 & $0.663-1.753$ & .762 \\
Race (non-Caucasian vs Caucasian) & 0.873 & $0.368-2.069$ & .757 \\
\hline$B A C$, Bronchioloalveolar carcinoma; $C I$, confidence interval; $C T$, computed tomogra- \\
phy; $C X R$, chest x-ray.
\end{tabular}

TABLE E4. Matched groups from propensity score analysis (C-statistic 0.640)

\begin{tabular}{lccc}
\hline \multicolumn{1}{c}{ Characteristic } & CT $(\mathbf{n}=\mathbf{1 7 4})$ & $\mathbf{C X R}(\mathbf{n}=\mathbf{1 7 4})$ & $\boldsymbol{P}$ value \\
\hline Age (mean $\pm \mathrm{SD})$ & $66 \pm 10$ & $68 \pm 10$ & .134 \\
Charlson index $($ mean $\pm \mathrm{SD})$ & $0.90 \pm 0.94$ & $0.89 \pm 1.01$ & .956 \\
T2a tumor & $56(32 \%)$ & $47(27 \%)$ & .291 \\
Resection (sublobar) & $33(19 \%)$ & $33(19 \%)$ & 1.0 \\
Adjuvant therapy & $10(6 \%)$ & $10(6 \%)$ & 1.0 \\
\hline
\end{tabular}

$C T$, Computed tomography; $C X R$, chest x-ray; $S D$, standard deviation.

\section{3.e2 The Journal of Thoracic and Cardiovascular Surgery • January 2015}


TABLE E5. Theoretic sample size calculation for a clinical trial of stage IA or stage IIIA non-small cell lung cancer based on 3-year accrual and additional 5-year follow-up

\begin{tabular}{lccccc}
$\begin{array}{l}\text { Median survival } \\
\text { in control group }\end{array}$ & Power & $\begin{array}{c}\text { Assumed improvement } \\
\text { in median survival }\end{array}$ & Sample size per group & Assuming 10\% attrition & Assuming 20\% attrition \\
\hline $119^{*}$ & 0.8 & $20 \%$ & 1380 & 1534 & 1725 \\
$119^{*}$ & 0.9 & $20 \%$ & 1847 & 2053 & 2309 \\
$23 \dagger$ & 0.8 & $20 \%$ & 464 & 516 & 580 \\
$23 \dagger$ & 0.9 & $20 \%$ & 621 & 690 & 863 \\
\hline
\end{tabular}

*Median survival pathologic stage IA NSCLC. †Median survival pathologic stage IIIA NSCLC. Median survival based on Goldstraw P, Crowley J, Chansky K, Giroux DJ, Groome PA, Rami-Porta R, et al. The IASLC Lung Cancer Staging Project: proposals for the revision of the TNM stage groupings in the forthcoming (seventh) edition of the TNM Classification of malignant tumours. J Thorac Oncol. 2007;2:706-14. 